\title{
Quantum phase transitions in a spin-1 antiferromagnetic chain with long-range interactions and modulated single-ion anisotropy
}

\author{
Jie Ren $\odot,{ }^{1}$ Wen-Long You $\odot,{ }^{2,3,}{ }^{*}$ and Andrzej M. Oleś $\odot^{4,5}$ \\ ${ }^{1}$ School of Electronic and Information Engineering, Changshu Institute of Technology, Changshu 215500, China \\ ${ }^{2}$ College of Science, Nanjing University of Aeronautics and Astronautics, Nanjing 211106, China \\ ${ }^{3}$ School of Physical Science and Technology, Soochow University, Suzhou, Jiangsu 215006, China \\ ${ }^{4}$ Institute of Theoretical Physics, Jagiellonian University, Profesora Stanisława Łojasiewicza 11, PL-30348 Kraków, Poland \\ ${ }^{5}$ Max Planck Institute for Solid State Research, Heisenbergstrasse 1, D-70569 Stuttgart, Germany
}

(Received 19 May 2020; accepted 6 July 2020; published 17 July 2020)

\begin{abstract}
We study the phase diagram of a spin-1 antiferromagnetic chain with isotropic antiferromagnetic interactions decaying with a power law $\propto r^{-\alpha}(\alpha \geqslant 1)$ accompanied by modulated single-ion anisotropy. Employing the techniques of the density-matrix renormalization group, the effects of long-range interactions and singleion anisotropy on a variety of correlations are investigated. In order to check the consistency, the fidelity susceptibilities are evaluated across quantum phase transitions. The quantum critical points are faithfully detected and orders of phase transitions are determined. The correlation-length critical exponent is extracted from scaling functions of the fidelity susceptibility. The presence of long-range interactions leads to a quantitative change of the phase boundaries and reduces the order of the phase transition under certain conditions. A direct first-order transition between the periodic Néel phase and the large- $D$ phase occurs for slowly decaying antiferromagnetic interactions.
\end{abstract}

DOI: 10.1103/PhysRevB.102.024425

\section{INTRODUCTION}

The Haldane phase [1,2] of the one-dimensional (1D) antiferromagnetic (AF) spin-1 $(S=1)$ chain has received continued attention as it is closely related to the breaking of a hidden $\mathbb{Z}_{2} \otimes \mathbb{Z}_{2}$ symmetry [3] through a nonlocal unitary transformation. In fact, long-range order is absent in the isotropic 1D spin-1 chain with a short-range exchange interaction but the ground state is characterized by a finite spectral gap and exponentially decaying AF spin correlations. The thoroughly studied gapped phase was conjectured by Haldane [1,2], and was confirmed in a series of experimental and theoretical papers [4-8]. An important benchmark of the Haldane phase is the occurrence of the nonlocal string order in the isotropic AF $S=1$ Heisenberg chain [9]. At the same time, the string order is a manifestation of the topological hidden order, as pointed out by Kennedy and Tasaki [3].

Frustration may be introduced in the AF spin-1 chain by other interactions, and as a result novel types of order could emerge. For instance, Affleck, Lieb, Kennedy, and Tasaki (AKLT) proposed [10] an extended model of an $S=1$ chain with an additional biquadratic spin interaction, whose ground

\footnotetext{
*wlyou@nuaa.edu.cn
}

Published by the American Physical Society under the terms of the Creative Commons Attribution 4.0 International license. Further distribution of this work must maintain attribution to the author(s) and the published article's title, journal citation, and DOI. Open access publication funded by the Max Planck Society. state is exactly solvable and gives the phase depicted in Fig. 1(a). The fourfold ground-state degeneracy arises due to two free edge $S=1 / 2$ spins for a chain with an open
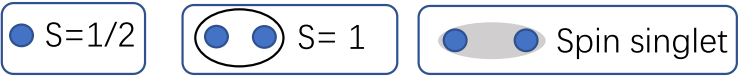

(a)

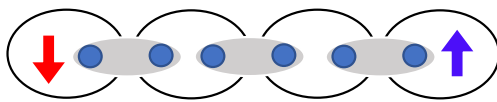

Haldane

(b)

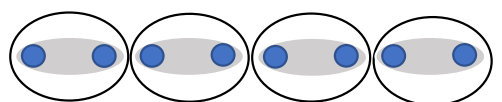

Large- $D$

(C)

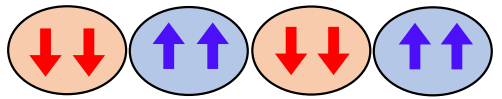

Néel

(d)

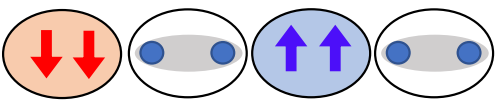

Periodic Néel

FIG. 1. Schematic view of various phases considered in this paper for the 1D AF $S=1$ chain. Each spin $S=1$ consists of two $S=1 / 2$ spins (blue dots); a pair of spins built out of two adjacent spins and connected by a gray ellipse forms a singlet, $(|\uparrow \downarrow\rangle-|\downarrow \uparrow\rangle) / \sqrt{2}$. Depending on the actual parameters, the possible phases of a frustrated 1D AF $S=1$ chain investigated here are as follows: (a) the Haldane phase (the AKLT state) which has two free edge $S=1 / 2$ spins for an open boundary condition; (b) the singlet nondegenerate (large- $D$ ) state for a sufficiently large singlesite anisotropy $D_{1}>0$; (c) the usual $S=1$ Néel phase, $|\uparrow \downarrow \uparrow \downarrow\rangle$; and (d) the periodic Néel phase which includes $|0\rangle$ states as separating $|\uparrow\rangle$ and $|\downarrow\rangle$ states, i.e., $|\uparrow 0 \downarrow 0\rangle$. 
boundary condition and provides crucial insight into the symmetry-protected topological (SPT) order. Such a general bilinear-biquadratic Hamiltonian leads to a dimerized spin-1 chain for a range of parameters [10]. Note that the biquadratic spin-spin interaction, $\propto\left(\mathbf{S}_{i} \cdot \mathbf{S}_{i+1}\right)^{2}$, arises naturally within the $S=1$ Heisenberg model describing the strong-coupling theory of the iron pnictides [11-13].

Yet, perhaps the simplest frustrated model for the $\mathrm{AF}$ spin-1 chain includes both AF nearest-neighbor and nextnearest-neighbor interactions-it has been shown [14] that the latter dilutes the AKLT phase by singlet bond correlations [15]. Here, we go further and analyze the frustrated spin-1 chain with infinite-range AF correlations which decay algebraically in the presence of a superposition of uniform and alternating uniaxial single-ion anisotropy. We show below that such a model gives an interesting competition of spin correlations with those encountered in the Haldane phase.

Experimentally, the Haldane chain was most comprehensively studied via inelastic neutron scattering in $S=1$ chain materials, such as $\mathrm{SrNi}_{2} \mathrm{~V}_{2} \mathrm{O}_{8}$ [16,17] and $\mathrm{Ni}\left(\mathrm{C}_{2} \mathrm{H}_{8} \mathrm{~N}_{2}\right)_{2} \mathrm{NO}_{2}\left(\mathrm{ClO}_{4}\right)$ [18]. To reproduce the experimental findings for real materials, additional terms have to be added to the ideal Heisenberg Hamiltonian, such as exchange anisotropy, bond alternation, or single-ion anisotropy. Anisotropy effects can then significantly modify the groundstate magnetic properties. A sufficiently strong easy-plane single-ion anisotropy $\propto D\left(S_{i}^{z}\right)^{2}$ induces a Gaussian quantum phase transition (QPT) with central charge $c=1$ from the SPT state to a topologically trivial large- $D$ phase [19-25] [cf. Fig. 1(b)], such as the one encountered in $\mathrm{NiCl}_{2} 4 \mathrm{SC}\left(\mathrm{NH}_{2}\right)_{2}$ (DTN) [26,27].

Recently, artificial materials have been adopted to simulate quasi-1D quantum materials in atomic, molecular, and optical systems [28-30]. Different power-law decays of longrange $\mathrm{AF}$ exchange interactions were considered in quasi-1D quantum chains, such as the Coulomb-like interaction $\propto 1 / r$ [31], the dipole-dipole interaction $\propto 1 / r^{3}$ [32-34], and the van der Waals interaction $\propto 1 / r^{6}$ [31]. More recently, power-lawdecaying long-range interactions $\propto 1 / r^{\alpha}$ were also realized in which the power $\alpha \geqslant 1$ could even be continuously adjusted in some region using careful manipulation [34-40].

We emphasize that long-range interactions cannot be considered as perturbations and play an essential role in the critical phenomena [41-49]. Recently, two of us considered a chain with anisotropic long-range-decaying interactions and investigated its QPTs [50]. Interesting phase diagrams were established, with the long-range interactions of the $z$ component resulting in a Wigner crystal phase, and the transversal one resulting in a symmetry broken phase. The present work addresses primarily the QPTs in the spin- 1 chains with long-range interactions and modulated single-ion anisotropy. Using a combination of density-matrix renormalization group (DMRG) calculations and a spin-wave analysis, various correlations for a spin-1 chain with long-range interactions were presented. The concept of fidelity susceptibility has been successfully used to classify QPTs in the spin-1 AF chain in the past [24,25,51-59]. We employ here the fidelity susceptibility and establish a rich phase diagram of a spin-1 chain by changing the uniform and alternating single-ion anisotropy.
The remainder of this paper is organized as follows. We introduce the $S=1$ Heisenberg model with long-range interactions and alternating single-ion anisotropy in Sec. II. The details of the numerical methods and information metric are also introduced. In Sec. III, the effects of long-range interactions and modulated single-ion anisotropy are investigated. Using fidelity susceptibility, the phase diagrams for various cases are determined. The characteristics of each phase are interpreted by diverse correlations and effective Hamiltonians in various limiting cases. A discussion and summary are presented in Sec. IV.

\section{HAMILTONIAN AND CORRELATIONS}

\section{A. Frustrated Heisenberg chain}

The Hamiltonian of the spin-1 Heisenberg chain of length of $N$ sites with long-range interactions and modulated singleion anisotropy is given by

$$
H=\sum_{i<j}^{N} J_{i j} \mathbf{S}_{i} \cdot \mathbf{S}_{j}+\sum_{i=1}^{N}\left[D_{1}+(-1)^{i} D_{2}\right]\left(S_{i}^{z}\right)^{2},
$$

where $\mathbf{S}_{i}$ is the spin-1 operator on site $i$. We consider spin states using the following notation $|\uparrow\rangle,|0\rangle$, and $|\downarrow\rangle$ for the single-site states with $S_{i}^{z}=1,0$, and -1 , respectively. The interactions $J_{i j}$ between two spins decay algebraically with distance $r=|i-j| \geqslant 1$, i.e., as

$$
J_{i, i+r}=J r^{-\alpha},
$$

and we take $\alpha \geqslant 1$. The parameters $D_{1}$ and $D_{2}$ stand for uniform and alternating components of uniaxial single-ion anisotropy, respectively. For convenience, we take $J=1$ for nearest neighbors $(|i-j| \equiv 1)$ and use open boundary conditions in this study. Note that for $D_{1}=D_{2}$ one finds single-ion anisotropy only at every second site.

\section{B. Order parameter and spin correlations}

According to the Ginzburg-Landau scenario, a welldefined order parameter is a vital ingredient for characterizing the nature of a phase. In order to characterize the QPTs, the two-point correlations

$$
C_{l, m}^{\alpha}=\left\langle S_{l}^{\alpha} S_{m}^{\alpha}\right\rangle \quad(\alpha=x, y, z)
$$

and the nonlocal string order parameter (SOP),

$$
O_{l, m}^{z}=-\left\langle S_{l}^{z} \exp \left(i \pi \sum_{k=l+1}^{m-1} S_{k}^{z}\right) S_{m}^{z}\right\rangle,
$$

are defined. The difficulty in defining suitable order parameters in miscellaneous phases therein motivates us to adopt instead widely accepted information measures.

As a Riemannian metric in the parameter space, quantum fidelity susceptibility is intimately related to quantum fluctuations and dissipative responses of the system. Consider the Hamiltonian $H(\lambda)$ with a set of external parameters, $\lambda=\left\{\lambda_{1}, \lambda_{2}, \ldots, \lambda_{\kappa}\right\}$, where $\kappa$ is the dimension of the parameter space. The quantum geometric tensor describes the geometric structure upon projecting the dynamics onto the 

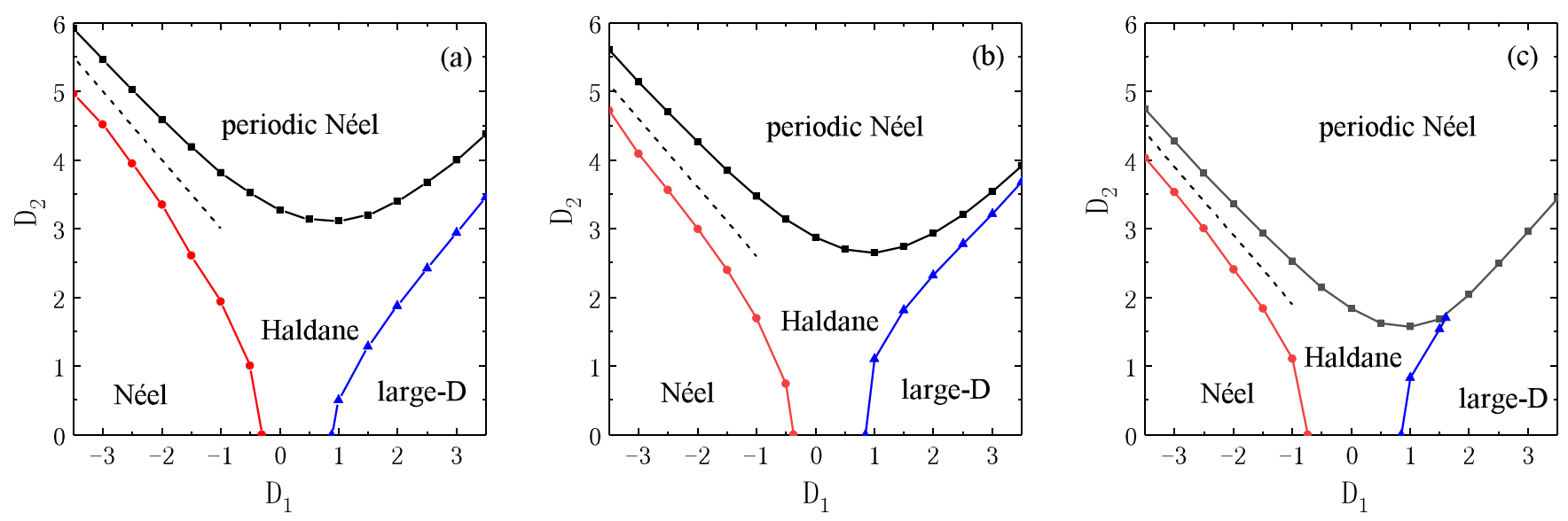

FIG. 2. Phase diagram of the spin-1 AF chain Eq. (1) as in the $\left\{D_{1}, D_{2}\right\}$ plane with different $\alpha$. The locations of transition lines are obtained for a system size of $N=100$ sites, where we do not find any finite-size effects. The dashed lines in (a)-(c) separate approximately Néel from the periodic Néel phase; they are given by $D_{2}=-D_{1}+c_{0}$, with $c_{0}$ selected for a given value of $\alpha$. Parameters are (a) $\alpha=10, c_{0} \simeq 2.0$, (b) $\alpha=3, c_{0} \simeq 1.6$, and (c) $\alpha=1, c_{0} \simeq 0.95$.

(nondegenerate) ground state $\psi_{0}(\boldsymbol{\lambda})$, given by

$$
\chi_{\mu, \nu}=\left\langle\partial_{\mu} \psi_{0}(\lambda)\left(1-\left|\psi_{0}(\lambda)\right\rangle\left\langle\psi_{0}(\lambda)\right|\right) \mid \partial_{\nu} \psi_{0}(\lambda)\right\rangle .
$$

The imaginary part of the geometric tensor $\operatorname{Im}\left\{\chi_{\mu, \nu}\right\}$ is related to the Berry curvature, and the real part $\operatorname{Re}\left\{\chi_{\mu, \nu}\right\}$ is dubbed the quantum fidelity susceptibility which measures the change rate of the distance between the two closest states as a driving parameter $\lambda_{\mu}$ is modulated [60]. The quantum geometric tensor has been experimentally extracted by using a superconducting qubit [61], coupled qubits in diamond [62], and exciton polaritons in a planar microcavity [63].

As an information metric in the Hilbert space, quantum fidelity susceptibility has a gravity dual with the spatial volume of the Einstein-Rosen bridge in anti-de Sitter (AdS) space [64]. The sensitivity is greatly enhanced especially for the system at the quantum criticality comparing with that away from the critical region [65]. The divergence of $\chi_{\mu, \mu}$ (5) (in what follows we use an abbreviation $\chi$ for this quantity) can directly signal a QPT and locate the quantum critical points. Quantum fidelity susceptibility has been proved to play the role of a universal order parameter in identifying the QPTs [66-68].

Both of these observations require us to calculate the ground state or the reduced density matrix of the system. Based on matrix product states, the finite-size DMRG technique was adopted [69-71]. In the numerics we keep up to $m=300$ eigenstates during the procedure of basis truncation and the number of sweeps is $n=50$. These conditions guarantee that the simulation converges sufficiently fast and the truncation error is smaller than $10^{-9}$.

\section{NUMERICAL RESULTS}

\section{A. Phase diagrams}

First, we performed calculations for varying uniaxial single-ion anisotropy parametrized by $D_{1}$ and $D_{2}$ [see Eq. (1)] with fixed values of $\alpha=10,3$, and 1 , respectively. One finds then generic phase diagrams, with the Néel (large- $D$ ) phase for a negative (positive) value of $D_{1}$ and the Haldane phase in between (see Fig. 2). A large (positive) value of $D_{2}$ induces a periodic Néel state. The characteristic spin states are visualized for different phases in Fig. 1. One finds always four phases; their range of stability changes when the exponent $\alpha$, which describes the long-range decay, varies from $\alpha=\infty$ (the AF Heisenberg model with nearest-neighbor AF interactions) to $\alpha=1$. However, the intermediate Haldane phase shrinks as $\alpha$ decreases.

Remarkably, for the AF Heisenberg model with nearestneighbor interactions (not shown for a general case), numerical studies revealed that the Haldane phase creeps in between the periodic Néel phase, the Néel phase, and the large- $D$ phase. This limit is reached with an increasing value of $\alpha$ from a finite value - then the Haldane phase is squeezed, and a direct QPT from the large- $D$ phase to the periodic Néel phase occurs for large $D_{1}$ through a first-order QPT. The critical line of the periodic Néel to large- $D$ transition will occur at $D_{1}=D_{2}$ for sufficiently large single-ion anisotropy $D_{1}$.

A tricritical point emerges at moderate $D_{1}$ for $\alpha=1$ [see Fig. 2(c)]. However, there is still no direct transition between the Néel phase and the periodic Néel phase, which emerges from the Haldane state by lowering specific Néel-type spin configurations compatible with the spatial modulation of single-site anisotropy. A narrow Haldane phase would survive in the neighborhood of the line $D_{1}+D_{2}=c_{0}$, i.e., at $D_{2}=-D_{1}+c_{0}$, where $c_{0}$ is a constant and depends on the parameter $\alpha$.

To better understand such a rich phase diagram of Fig. 2 that results from the interplay of long-range AF couplings and alternating single-ion anisotropies, we first consider the cases with either $D_{2}=0$ or $D_{1}=0$ (see Fig. 3). For $\alpha=\infty$ (the AF Heisenberg model), the system reduces to a spin-1 chain with a nearest-neighbor interaction, and it is in the Haldane phase at the isotropic point $\left(D_{1}=0\right.$ and $\left.D_{2}=0\right)$, which is composed of the superposition of states with hidden nonlocal AF order. At $D_{2}=0$ one finds two QPTs by increasing the value of $D_{1}$, from the Néel phase through the Haldane phase to the large- $D$ phase [see Fig. 3(a)]. By going upwards vertically at $D_{1}=0$, there is only one QPT for increasing 

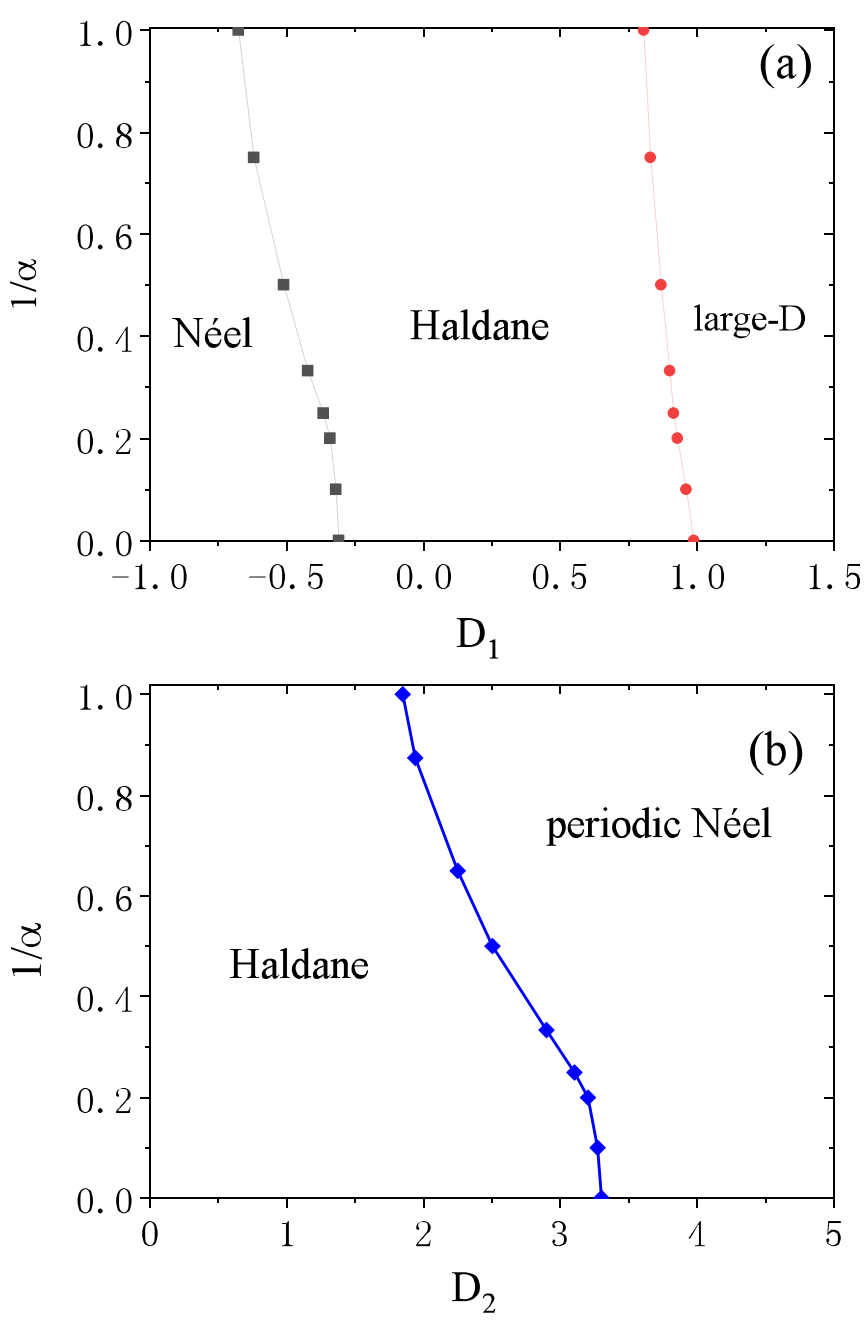

FIG. 3. Examples of QPTs found for the spin-1 chain in Fig. 2 as functions of $\alpha$ for (a) uniform anisotropy $D_{1}$, with $D_{2}=0$, and (b) modulated anisotropy $D_{2}$, with $D_{1}=0$.

$D_{2}$, from the Haldane phase to the periodic Néel phase [see Fig. 3(b)].

Figure 4 shows spin-spin correlation functions for the different phases found in the phase diagram of Fig. 2. In Fig. 4(a) one observes that both spin correlation functions, $C_{1, i}^{z}$ and $C_{1, i}^{x}$, vanish fast over a separation of just a few sites, while the nonlocal $O_{1, i}^{z}$ (4) converges quickly to $0.452(0.358)$ for odd (even) site $i$, manifesting the existence of the Haldane phase. The odd-even effect follows from the open boundary condition. At $D_{1}=2$ and $D_{2}=0$ one finds the large- $D$ phase [see Fig. 4(b)].

Some specific AF order will be favored by varying the single-site anisotropy. Changing the sign of $D_{1}$, one finds the Néel phase at $D_{1}=-2$ [see Fig. 4(c)]. The average magnetic moments are $\left\langle S_{1}^{z}\right\rangle \simeq 0.94$ for the edge site and $\left\langle S_{50}^{z}\right\rangle \simeq 0.90$ in the middle of the chain. On the other hand, for a quite large alternating single-site anisotropy, i.e., $D_{2} \gg J$, the spins on the odd sites are restricted to $\left\langle S_{2 i-1}^{z}\right\rangle \approx \pm 1$ and those on the even sites are confined to $\left\langle S_{2 i}^{z}\right\rangle=0$. In Fig. 4(d) this state is shown for $D_{1}=0$ and $D_{2}=4$. Indeed, two-spin correlations $C_{1, i}^{z}$ oscillate periodically between the values being close to $-1,0$, and 1 , and $C_{1, i}^{x} \approx 0$.

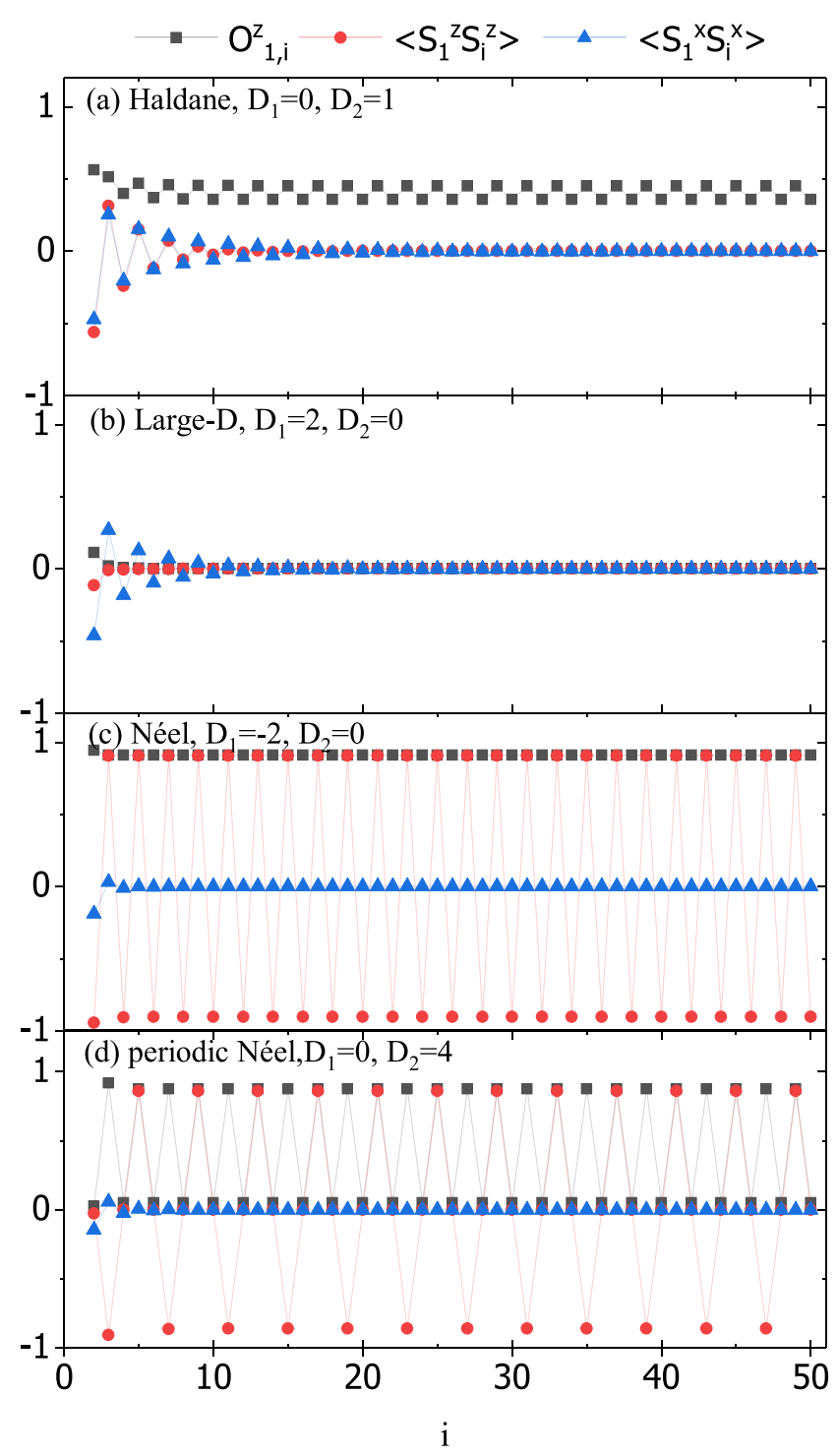

FIG. 4. Spin-spin correlation functions $\left\langle S_{l}^{\alpha} S_{m}^{\alpha}\right\rangle$, and string order parameter $O_{l, m}^{z}$ (4) as obtained with $N=100$ for four representative sets of $\left\{D_{1}, D_{2}\right\}$ parameters (see legends) which correspond to different phases of the spin-1 AF Heisenberg chain (with $\alpha=\infty$ ).

To elucidate the dominated configurations, the effective interactions between $S_{2 i-1}^{z}$ and $S_{2 i+1}^{z}$ as the first-order perturbation in $J$ plays the leading role, which for a chain of even length $N$ is of the form

$$
H_{\mathrm{eff}}^{(1)}=J_{13} \sum_{i=1}^{N / 2-1} S_{2 i-1}^{z} S_{2 i+1}^{z} .
$$

The crucial point here is the AF coupling $J_{13}$ between nextnearest-neighbor spins at odd sites which triggers the periodic Néel phase, being $|\cdots \uparrow 0 \downarrow 0 \uparrow 0 \downarrow\rangle \cdots\rangle$ [cf. Fig. 1(d)]; the long-range interactions frustrate this term. For an open system, one finds the net magnetization being zero if $(N-2)$ sites is a multiple of 4 , i.e., the considered open chain may accommodate a certain number of unit cells of length 

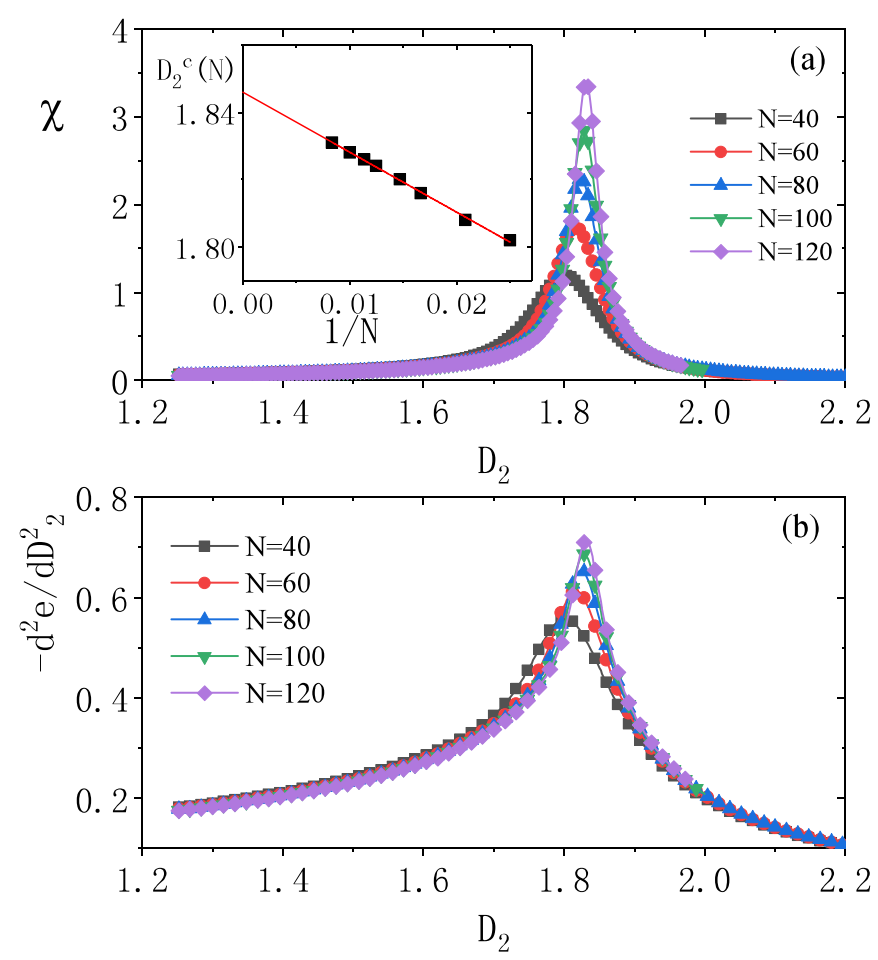

FIG. 5. (a) Fidelity susceptibility per site $\chi$ plotted as a function of the alternating anisotropy parameter $D_{2}$ for different system sizes (see legends). The inset shows the finite-size scaling of the maximum of the fidelity susceptibility $D_{2}^{c 1}$. The lines are fitted and represent guides to the eye. (b) The second derivative of the ground-state energy density $\left(d^{2} e / d D_{2}^{2}\right)$ plotted for increasing parameter $D_{2}$ for different system sizes. Parameters: $\alpha=1$ and $D_{1}=0$.

four. Quantum fluctuation corrections are small but somewhat larger than in the Néel phase, and one finds $\left|\left\langle S_{2 i-1}^{z}\right\rangle\right| \simeq 0.88$.

\section{B. Spin correlations and fidelity susceptibility}

The phase transition between the Haldane phase and the periodic Néel phase can be accurately determined by the analysis of the fidelity susceptibility. The results for $D_{1}=0$ and $\alpha=1$ are shown in Fig. 5(a). With increasing $D_{2}$, a peak of the ground-state fidelity susceptibility is observed at $D_{2}^{*} \simeq 1.84$, which signals an approach to the transition in the thermodynamic limit, $N \rightarrow \infty$. Further evidence for identifying the QPT is provided by the results of the second derivative of the ground-state energy density $\left(d^{2} e / d D_{2}^{2}\right)$, which is shown in Fig. 5(b). We thus confirm that the Haldane-to-periodicNéel transition is a QPT of second order.

According to the finite-size scaling theory [72], the position of the maximal points of the fidelity susceptibility can be fitted by the following formula,

$$
\left|D_{2}^{*}(N)-D_{2}^{c}\right| \sim N^{-b}
$$

where $b$ is a constant given by the critical exponent $v$, $b \equiv 1 / \nu$, and $D_{2}^{c}$ is the quantum critical point in the thermodynamic limit. Accordingly, the scaling of the extremal points of the fidelity susceptibility gives rise to $D_{2}^{c}=1.845, b_{1} \simeq 1$, as is shown in the inset of Fig. 5(a). For a second-order QPT, it is shown that the fidelity susceptibility at the peak point for
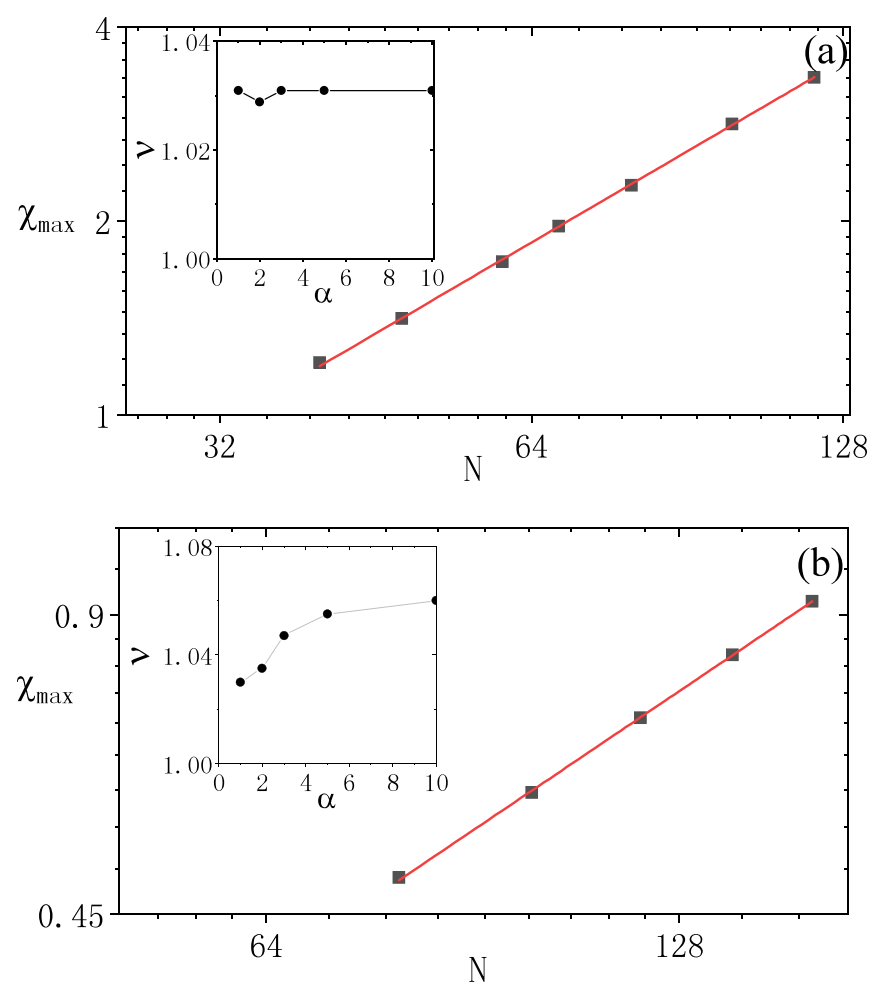

FIG. 6. Log-log plot of maximum fidelity susceptibility $\chi_{\max }$ as a function of the chain length $N$ with $\alpha=1$ for (a) the Haldane-toperiodic-Néel QPT with $D_{1}=0$, and (b) the Haldane-to-Néel QPT with $D_{2}=0$. The insets show the critical exponent $v$ as a function of the parameter $\alpha$ [see Eq. (2)].

finite size $N$ behaves as

$$
\chi\left(D_{2}^{*}\right) \propto N^{\mu-1}
$$

where $\mu$ is the critical adiabatic dimension.

The critical exponent $v$ of the correlation length can be obtained from $\mu, v=2 / \mu$. As is disclosed in Fig. 6(a), the linear dependence of the log-log plot suggests that $\mu=1.94$ and $v=1.03$. This illustrates that the Haldane-to-periodicNéel QPT belongs to the Ising universality class [73,74]. We recall that the phase diagram of the model Hamiltonian (1) for $D_{1}=0$ is shown in Fig. 3(a), and the whole critical line corresponds to second-order QPT. For the AF Heisenberg model (at $\alpha=\infty$ ), the Haldane-to-periodic-Néel transition occurs at $D_{2} \simeq 3.30[20,58]$. It is seen that the critical point $D_{2}^{c 1}$ drops when $\alpha$ decreases.

Analogously, we consider the uniform case with $D_{2}=0$, see Fig. 6(b). Although the AF long-range interactions are frustrated, the system would be reminiscent of a Haldane phase when $D_{1}=0$ and $\alpha \leqslant 1$ [47]. The system is in the Haldane phase for $\alpha=\infty$ and $-0.31<D_{1}<0.99$ $[19,21,25,51]$. For $D_{1} \ll-J$, all spins are restricted to be in the states $\left\langle S_{i}^{z}\right\rangle= \pm 1$. When $\alpha=\infty$, the effective coupling between the nearest-neighbor spins is obtained within the first-order perturbation theory in $J$ as [20]

$$
H_{\mathrm{eff}}^{(1)}=J_{12} \sum_{i} S_{i}^{z} S_{i+1}^{z} \text {. }
$$



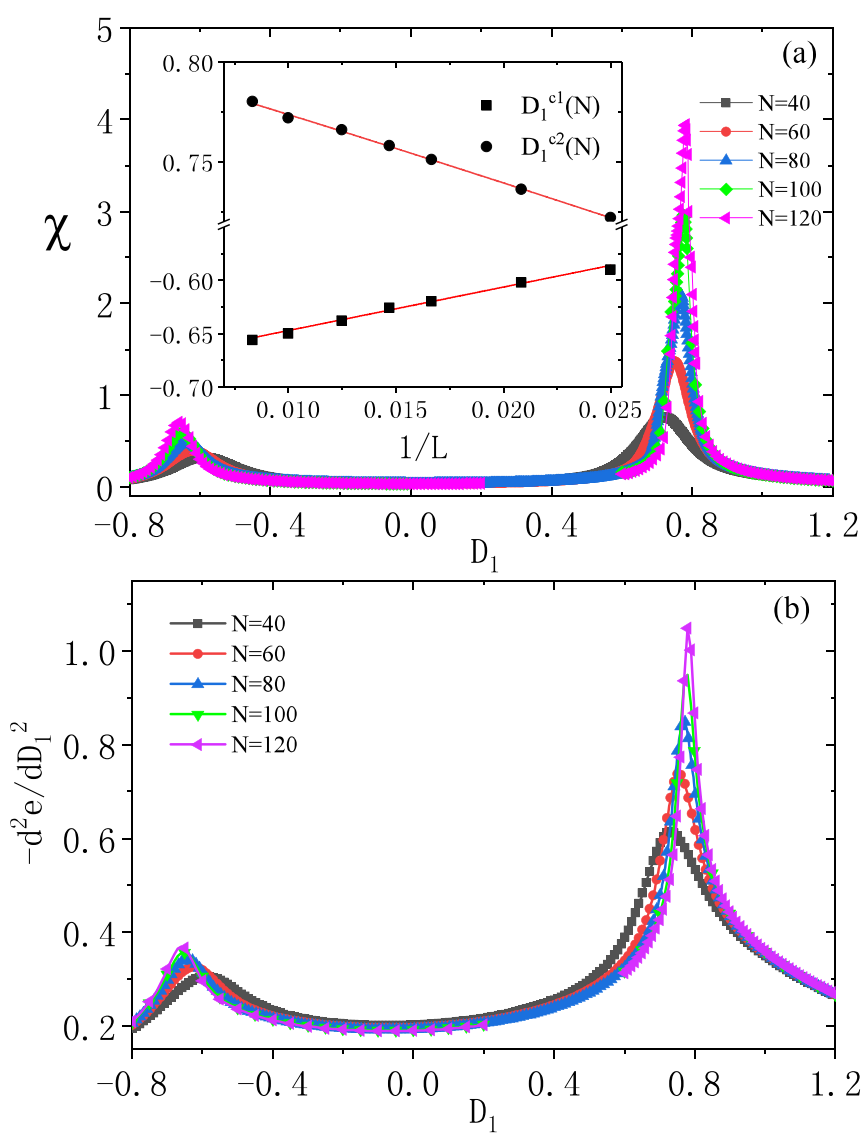

FIG. 7. (a) Fidelity susceptibility $\chi$ per site plotted as a function of the parameter $D_{1}$ for different system sizes, $N \in[40,120]$, with $\alpha=1$. The inset shows the finite-size scaling of $D_{1}^{c 1}$ and $D_{1}^{c 2}$ of the fidelity susceptibility. The lines are fitted straight lines. (b) The second derivative of the ground-state energy density plotted as a function of the parameter $D_{1}$ for different system sizes, $N \in[40,120]$, with $\alpha=1$.

In this regard, the system is in the $|\uparrow \downarrow \uparrow \downarrow\rangle$-type Néel phase for a strong easy-axis single-site anisotropy $D_{1}<-0.31$. In the Néel phase, the two-spin correlations $C_{1, i}^{z} \simeq(-1)^{i-1}$ and $C_{1, i}^{x}$ decay exponentially over the distance between two spins, as shown in Fig. 4(c). In the opposite limit, i.e., with $D_{1} \gg J$, spins will be confined to be in the state $\left|\left\langle S_{i}^{z}\right\rangle\right|=0$, annotating that the system enters the large- $D$ phase for a sufficiently large easy-plane anisotropy, $D_{1}>0.99$. In the large- $D$ phase, the correlations $C_{1, i}^{z}, C_{1, i}^{x}$, and $O_{1, i}^{z}$ vanish [see Fig. 4(b)].

In order to determine the phase boundary with high accuracy, we also calculated the fidelity susceptibility for $\alpha=1$ and $D_{2}=0$ of the ground state for a system size $N$ up to 120 . The ground-state fidelity susceptibility per site $\chi_{D_{1}, D_{1}} / N$ is plotted as a function of the parameter $D_{1}$ for different sizes $N$ in Fig. 7(a). Two peaks in the fidelity susceptibility can be seen and they both increase with increasing system size. This implies the divergence of fidelity susceptibility in the thermodynamic limit, which suggests the occurrence of two successive QPTs. We can conclude that the left peak indicates the Néel-to-Haldane transition and the right peak indicates the Haldane-to-large- $D$ transition. We then plot the location of the maximum fidelity susceptibility as a function of $1 / N$

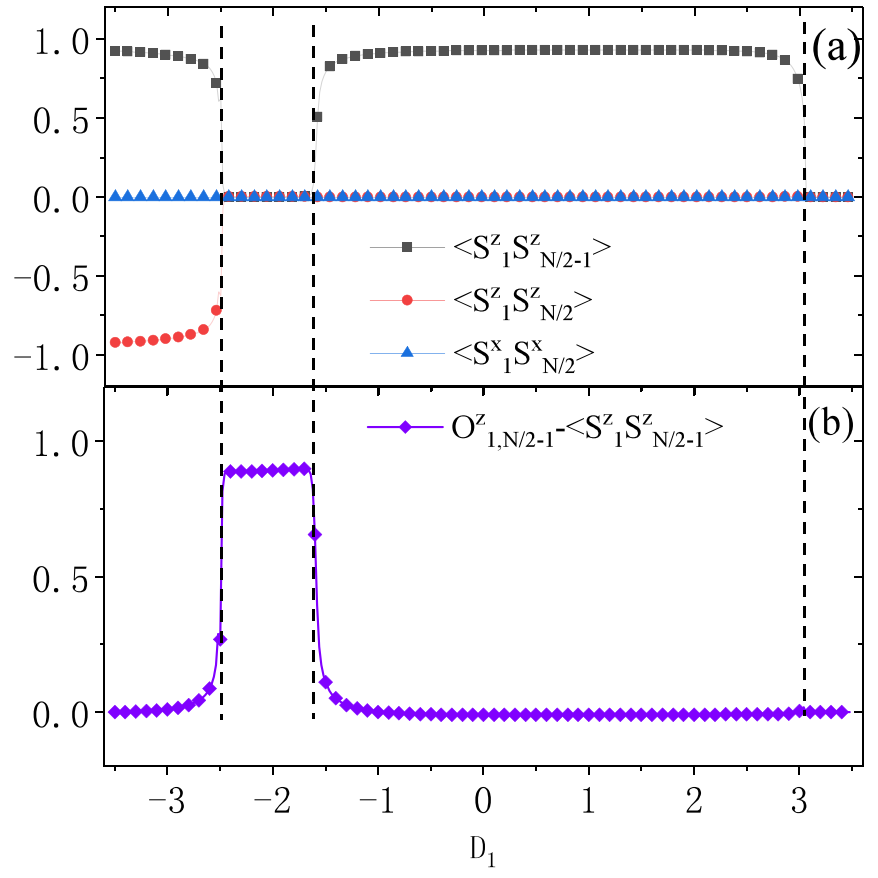

FIG. 8. Spin-spin correlation functions and string order parameter are plotted as functions of $D_{1}$ with $\alpha=1, D_{2}=3$. Parameter: $N=100$.

and show the numerical fits in the inset of Fig. 7(a). We obtain that $D_{1}^{c 1}=-0.688, b_{1} \simeq 1.05$, and $D_{1}^{c 2}=0.805, b_{2} \simeq 1.00$ according to Eq. (7).

Further evidence which indicates QPTs is provided by the second derivative of the ground-state energy density $\left(d^{2} e / d D_{1}^{2}\right)$ in Fig. 7(b). One observes that two peaks of $\left(d^{2} e / d D_{1}^{2}\right)$ become more pronounced for increasing system size $N$, which means that both of the phase transitions are of second order. As is shown in Fig. 6(b), the linear dependence of the log-log plot gives rise to $\mu=1.93$ and $v=1.03$ for the Haldane-to-Néel QPT with $\alpha=1, D_{2}=0$. The Haldane-toNéel phase transition belongs to the Ising universality class for all values of $\alpha$. However, it was reported that the Haldaneto-large- $D$ transition may be of higher order larger than two for the nearest-neighbor model $(\alpha=\infty)$ [25]. The long-range interaction would then reduce the order of the transition. The values of the critical points of Haldane-to-large- $D$ and Haldane-to-Néel shift to a lower value when $\alpha$ decreases, as is shown in Fig. 3(b).

After considering the special cases, we then speculate that both the easy-axis and easy-plane $D$ terms coexist in the Heisenberg chain. The correlation functions and the SOP for $D_{2}=3$ are plotted as a function of $D_{1}$ with $\alpha=1$ in Fig. 8(a). One finds $C_{1, N / 2-1}^{z} \simeq 1$ and $C_{1, N / 2}^{z} \simeq-1$ when $D_{1}<-2.5$. After surpassing the critical point, the $z$-component correlations vanish. When $D_{1}>-1.5$, the correlation $C_{1, N / 2-1}^{z}$ rebounds but $C_{1, N / 2}^{z}$ remains vanishing, which means that the system enters the periodic Néel phase. Furthermore, $C_{1, N / 2-1}^{z}$ vanishes suddenly at the periodic-Néel-to-large- $D$ transition point.

We also studied the SOP to characterize the phase in the range $-2.5<D_{1}<-1.5$ in Fig. $8(\mathrm{~b})$. It is noted that the SOP $O_{1, i}^{z} \approx\left|C_{1, i}^{z}\right|$ in the Néel phase. In order to determine the 
phase boundaries, we adopt $O_{1, N / 2-1}^{z}-\left|C_{1, N / 2-1}^{z}\right|$ to identify the Haldane phase, in which the long-distance correlation $C_{1, i}^{z}$ disappears in the Haldane phase [75].

\section{CONCLUSIONS}

In this paper, we investigated the quantum phase transitions in the one-dimensional spin-1 chains with long-range antiferromagnetic interactions decaying with a power law and modulated single-ion anisotropy by using the density-matrix renormalization group technique. Together with the shortrange correlations and the nonlocal string order parameter, the ground-state fidelity susceptibility was employed to determine the phase diagram and critical phenomena.

The presence of long-range interactions increased the difficulty of simulating the system numerically. Nevertheless, we provided compelling evidence for the phase transitions and critical lines in the thermodynamic limit. We identified four phases including the Haldane, large- $D$, the $|\uparrow \downarrow \uparrow \downarrow\rangle$ Néel phase, and the $|\uparrow 0 \downarrow 0\rangle$ periodic Néel phase. The appearance of long-range interactions modifies the phase boundaries and the order of the phase transition comparing with their counterparts with short-range interactions and leads to a direct first-order transition between periodic Néel and large- $D$ phase. However, a narrow Haldane phase survives between the periodic Néel and the Néel phase as the long-range interactions increase.

In summary, employing the scaling functions of the fidelity susceptibility gives a numerically economical way of obtaining accurately the correlation-length critical exponents.
We find that both the Haldane-to-periodic-Néel and Haldaneto-Néel quantum phase transitions are of second order and are classified for the model in Eq. (1) as belonging to the Ising universality class. The local correlations are capable of characterizing those phases as topological trivial ones, while only the nonlocal string order parameter can identify the topological phase. More precisely, the difference between the string order parameter and the two-point spin correlation, $O_{1, N / 2-1}^{z}-\left|C_{1, N / 2-1}^{z}\right|$, can exclusively detect the Haldane phase. We remark that the order of the Haldane-to-large- $D$ transition is higher than two for the nearest-neighbor model $(\alpha=\infty)$ and will drop to two as $\alpha$ decreases. Further studies of the spin ordered phases in the presence of long-range interactions is experimentally challenging and could also bring about some novel types of phase transitions.

\section{ACKNOWLEDGMENTS}

It is our pleasure to acknowledge insightful discussions with Marek Rams and Gaoyong Sun. This work is supported by the National Natural Science Foundation of China (NSFC) under Grants No. 11104021 and No. 11474211, as well as by the National Science Centre (NCN, Poland) under Project No. 2016/23/B/ST3/00839. W.-L.Y. acknowledges support by the startup fund (Grant No. 1008-YAH20006) of Nanjing University of Aeronautics and Astronautics. A.M.O. is grateful for the Alexander von Humboldt Foundation Fellowship (Humboldt-Forschungspreis).
[1] F. D. M. Haldane, Continuum dynamics of the 1-D Heisenberg antiferromagnet: Identification with the $\mathrm{O}(3)$ nonlinear sigma model, Phys. Lett. A 93, 464 (1983).

[2] F. D. M. Haldane, Nonlinear Field Theory of Large-Spin Heisenberg Antiferromagnets: Semiclassically Quantized Solitons of the One-Dimensional Easy-Axis Néel State, Phys. Rev. Lett. 50, 1153 (1983).

[3] T. Kennedy and H. Tasaki, Hidden $Z_{2} \times Z_{2}$ symmetry breaking in Haldane-gap antiferromagnets, Phys. Rev. B 45, 304 (1992); Hidden symmetry breaking and the Haldane phase in $S=1$ quantum spin chains, Commun. Math. Phys. 147, 431 (1992).

[4] M. den Nijs and K. Rommelse, Preroughening transitions in crystal surfaces and valence-bond phases in quantum spin chains, Phys. Rev. B 40, 4709 (1989).

[5] C. Degli Esposti Boschi, E. Ercolessi, F. Ortolani, and M. Roncaglia, On $c=1$ critical phases in anisotropic spin-1 chains, Eur. Phys. J. B 35, 465 (2003).

[6] K. Nomura, Spin-correlation functions of the $S=1$ Heisenberg-Ising chain by the large-cluster-decomposition Monte Carlo method, Phys. Rev. B 40, 9142 (1989).

[7] W. J. L. Buyers, R. M. Morra, R. L. Armstrong, M. J. Hogan, P. Gerlach, and K. Hirakawa, Experimental Evidence for the Haldane Gap in a Spin-1 Nearly Isotropic, Antiferromagnetic Chain, Phys. Rev. Lett. 56, 371 (1986).

[8] A. L. Malvezzi, G. Karpat, B. Çakmak, F. F. Fanchini, T. Debarba, and R. O. Vianna, Quantum correlations and coherence in spin-1 Heisenberg chains, Phys. Rev. B 93, 184428 (2016).
[9] S. R. White and D. A. Huse, Numerical renormalization-group study of low-lying eigenstates of the antiferromagnetic $S=1$ Heisenberg chain, Phys. Rev. B 48, 3844 (1993).

[10] I. Affleck, T. Kennedy, E. H. Lieb, and H. Tasaki, Rigorous Results on Valence-Bond Ground States in Antiferromagnets, Phys. Rev. Lett. 59, 799 (1987).

[11] D. Stanek, O. P. Sushkov, and G. S. Uhrig, Self-consistent spinwave theory for a frustrated Heisenberg model with biquadratic exchange in the columnar phase and its application to iron pnictides, Phys. Rev. B 84, 064505 (2011).

[12] R. Yu, Z. Wang, P. Goswami, A. H. Nevidomskyy, Q. Si, and E. Abrahams, Spin dynamics of a $J_{1}-J_{2}-K$ model for the paramagnetic phase of iron pnictides, Phys. Rev. B 86, 085148 (2012).

[13] Y. Wang, W. Hu, R. Yu, and Q. Si, Broken mirror symmetry, incommensurate spin correlations, and $B_{2 g}$ nematic order in iron pnictides, Phys. Rev. B 100, 100502 (2019).

[14] A. Kolezhuk, R. Roth, and U. Schollwöck, First Order Transition in the Frustrated Antiferromagnetic Heisenberg $S=1$ Quantum Spin Chain, Phys. Rev. Lett. 77, 5142 (1996); Variational and density-matrix renormalization-group studies of the frustrated antiferromagnetic Heisenberg $S=1$ quantum spin chain, Phys. Rev. B 55, 8928 (1997).

[15] J. H. Pixley, A. Shashi, and A. H. Nevidomskyy, Frustration and multicriticality in the antiferromagnetic spin-1 chain, Phys. Rev. B 90, 214426 (2014).

[16] A. K. Bera, B. Lake, A. T. M. N. Islam, B. Klemke, E. Faulhaber, and J. M. Law, Field-induced magnetic ordering 
and single-ion anisotropy in the quasi-one-dimensional Haldane chain compound $\mathrm{SrNi}_{2} \mathrm{~V}_{2} \mathrm{O}_{8}$ : A single-crystal investigation, Phys. Rev. B 87, 224423 (2013).

[17] A. K. Bera, B. Lake, A. T. M. N. Islam, O. Janson, H. Rosner, A. Schneidewind, J. T. Park, E. Wheeler, and S. Zander, Consequences of critical interchain couplings and anisotropy on a Haldane chain, Phys. Rev. B 91, 144414 (2015).

[18] T. Delica, K. Kopinga, H. Leschke, and K. K. Mon, Thermal properties of chains of antiferromagnetically coupled spins with $s=1$ : Numerical evidence of the Haldane gap at nonzero temperatures, Europhys. Lett. 15, 55 (1991).

[19] W. Chen, K. Hida, and B. C. Sanctuary, Ground-state phase diagram of $S=1 \mathrm{XXZ}$ chains with uniaxial single-ion-type anisotropy, Phys. Rev. B 67, 104401 (2003).

[20] K. Hida and W. Chen, Emergence of long period antiferromagnetic orders from Haldane phase in $S=1$ Heisenberg chains with $D$-modulation, J. Phys. Soc. Jpn. 74, 2090 (2005).

[21] L. Campos Venuti, C. Degli Esposti Boschi, E. Ercolessi, G. Morandi, F. Ortolani, S. Pasini, and M. Roncaglia, Stable particles in anisotropic spin-1 chains, Eur. Phys. J. B 53, 11 (2006).

[22] Y. C. Tzeng, H. Onishi, T. Okubo, and Y. J. Kao, Quantum phase transitions driven by rhombic-type single-ion anisotropy in the $S=1$ Haldane chain, Phys. Rev. B 96, 060404(R) (2017).

[23] M. T. Batchelor, X. W. Guan, and N. Oelkers, Thermal and magnetic properties of spin-1 magnetic chain compounds with large single-ion and in-plane anisotropies, Phys. Rev. B 70, 184408 (2004).

[24] J. Ren, Y. M. Wang, and W. L. You, Quantum phase transitions in spin-1 XXZ chains with rhombic single-ion anisotropy, Phys. Rev. A 97, 042318 (2018).

[25] Y. C. Tzeng, H. H. Hung, Y. C. Chen, and M. F. Yang, Fidelity approach to Gaussian transitions, Phys. Rev. A 77, 062321 (2008).

[26] V. S. Zapf, D. Zocco, B. R. Hansen, M. Jaime, N. Harrison, C. D. Batista, M. Kenzelmann, C. Niedermayer, A. Lacerda, and A. Paduan-Filho, Bose-Einstein Condensation of $S=1$ Nickel Spin Degrees of Freedom in $\mathrm{NiCl}_{2}-4 \mathrm{SC}\left(\mathrm{NH}_{2}\right)_{2}$, Phys. Rev. Lett. 96, 077204 (2006).

[27] S. A. Zvyagin, J. Wosnitza, C. D. Batista, M. Tsukamoto, N. Kawashima, J. Krzystek, V. S. Zapf, M. Jaime, N. F. Oliveira, and A. Paduan-Filho, Magnetic Excitations in the Spin-1 Anisotropic Heisenberg Antiferromagnetic Chain System $\mathrm{NiCl}_{2}-4 \mathrm{SC}\left(\mathrm{NH}_{2}\right)_{2}$, Phys. Rev. Lett. 98, 047205 (2007).

[28] I. Bloch, J. Dalibard, and W. Zwerger, Many-body physics with ultracold gases, Rev. Mod. Phys. 80, 885 (2008).

[29] K. Kim, M. S. Chang, S. Korenblit, R. Islam, E. E. Edwards, J. K. Freericks, G. D. Lin, L. M. Duan, and C. Monroe, Quantum simulation of frustrated Ising spins with trapped ions, Nature (London) 465, 590 (2010).

[30] I. Bloch, J. Dalibard, and S. Nascimbène, Quantum simulations with ultracold quantum gases, Nat. Phys. 8, 267 (2012).

[31] M. Saffman, T. G. Walker, and K. Mølmer, Quantum information with Rydberg atoms, Rev. Mod. Phys. 82, 2313 (2010).

[32] X. L. Deng, D. Porras, and J. I. Cirac, Effective spin quantum phases in systems of trapped ions, Phys. Rev. A 72, 063407 (2005)

[33] B. Yan, S. A. Moses, B. Gadway, J. P. Covey, K. R. A. Hazzard, A. M. Rey, D. S. Jin, and Jun Ye, Realizing a lattice spin model with polar molecules, Nature (London) 501, 521 (2013).
[34] T. Lahaye, C. Menotti, L. Santos, M. Lewenstein, and T. Pfau, The physics of dipolar bosonic quantum gases, Rep. Prog. Phys. 72, 126401 (2009).

[35] J. W. Britton, B. C. Sawyer, A. C. Keith, C.-C. J. Wang, J. K. Freericks, H. Uys, M. J. Biercuk, and J. J. Bollinger, Engineered two-dimensional Ising interactions in a trapped-ion quantum simulator with hundreds of spins, Nature (London) 484, 489 (2012).

[36] C. Schneider, D. Porras, and T. Schaetz, Experimental quantum simulations of many-body physics with trapped ions, Rep. Prog. Phys. 75, 024401 (2012).

[37] A. Browaeys, D. Barredo, and T. Lahaye, Experimental investigations of dipole-dipole interactions between a few Rydberg atoms, J. Phys. B: At., Mol. Opt. Phys 49, 152001 (2016).

[38] R. Islam, C. Senkol, W. C. Campbell, S. Korenblit, J. Smith, A. Lee, E. E. Edwards, C.-C. J. Wang, J. K. Freericks, and C. Monroe, Emergence and frustration of magnetism with variable-range interactions in a quantum simulator, Science 340, 583 (2013).

[39] P. Richerme, Z.-X. Gong, A. Lee, C. Senko, J. Smith, M. FossFeig, S. Michalakis, A. V. Gorshkov, and C. Monroe, Non-local propagation of correlations in quantum systems with long-range interactions, Nature (London) 511, 198 (2014).

[40] P. Jurcevic, B. P. Lanyon, P. Hauke, C. Hempel, P. Zoller, R. Blatt, and C. F. Roos, Quasiparticle engineering and entanglement propagation in a quantum many-body system, Nature (London) 511, 202 (2014).

[41] W. Dür, L. Hartmann, M. Hein, M. Lewenstein, and H.-J. Briegel, Entanglement in Spin Chains and Lattices with Long-Range Ising-Type Interactions, Phys. Rev. Lett. 94, 097203 (2005).

[42] T. Koffel, M. Lewenstein, and L. Tagliacozzo, Entanglement Entropy for the Long-Range Ising Chain in a Transverse Field, Phys. Rev. Lett. 109, 267203 (2012).

[43] G. Sun, Fidelity susceptibility study of quantum long-range antiferromagnetic Ising chain, Phys. Rev. A 96, 043621 (2017).

[44] Z. Zhu, G. Sun, W.-L. You, and D. N. Shi, Fidelity and criticality of a quantum Ising chain with long-range interactions, Phys. Rev. A 98, 023607 (2018).

[45] Z.-X. Gong, M. F. Maghrebi, A. Hu, M. L. Wall, M. FossFeig, and A. V. Gorshkov, Topological phases with long-range interactions, Phys. Rev. B 93, 041102(R) (2016).

[46] M. F. Maghrebi, Z.-X. Gong, and A. V. Gorshkov, Continuous Symmetry Breaking in 1D Long-Range Interacting Quantum Systems, Phys. Rev. Lett. 119, 023001 (2017).

[47] Z. X. Gong, M. F. Maghrebi, A. Hu, M. Foss-Feig, P. Richerme, C. Monroe, and A. V. Gorshkov, Kaleidoscope of quantum phases in a long-range interacting spin-1 chain, Phys. Rev. B 93, 205115 (2016).

[48] Z. X. Gong, M. Foss-Feig, F. G. S. L. Brandão, and A. V. Gorshkov, Entanglement Area Laws for Long-Range Interacting Systems, Phys. Rev. Lett. 119, 050501 (2017).

[49] I. Frérot, P. Naldesi, and T. Roscilde, Entanglement and fluctuations in the XXZ model with power-law interactions, Phys. Rev. B 95, 245111 (2017).

[50] J. Ren, W.-L. You, and X. Wang, Entanglement and correlations in a one-dimensional quantum spin- $\frac{1}{2}$ chain with anisotropic power-law long-range interactions, Phys. Rev. B 101, 094410 (2020). 
[51] Y. C. Tzeng and M. F. Yang, Scaling properties of fidelity in the spin-1 anisotropic model, Phys. Rev. A 77, 012311 (2008).

[52] J. Ren and S. Q. Zhu, Quantum phase transition in easy-axis antiferromagnetic Heisenberg spin-1 chain, Phys. Rev. A 79, 034302 (2009).

[53] D. Schwandt, F. Alet, and S. Capponi, Quantum Monte Carlo Simulations of Fidelity at Magnetic Quantum Phase Transitions, Phys. Rev. Lett. 103, 170501 (2009).

[54] A. F. Albuquerque, F. Alet, C. Sire, and S. Capponi, Quantum critical scaling of fidelity susceptibility, Phys. Rev. B 81, 064418 (2010).

[55] V. Gritsev and A. Polkovnikov, in Understanding Quantum Phase Transitions, edited by L. D. Carr (Taylor \& Francis, Boca Raton, FL, 2010).

[56] M. M. Rams and B. Damski, Quantum Fidelity in the Thermodynamic Limit, Phys. Rev. Lett. 106, 055701 (2011); Scaling of ground-state fidelity in the thermodynamic limit: XY model and beyond, Phys. Rev. A 84, 032324 (2011).

[57] Y. H. Su, S. Y. Cho, Bo Li, H.-L. Wang, and H.-Q. Zhou, Non-local correlations in the Haldane phase for an XXZ spin-1 chain: A perspective from infinite matrix product state representation, J. Phys. Soc. Jpn. 81, 074003 (2012).

[58] J. Ren, G. H. Liu, and W. L. You, Entanglement entropy and fidelity susceptibility in the one-dimensional spin-1 XXZ chains with alternating single-site anisotropy, J. Phys.: Condens. Matter 27, 105602 (2015).

[59] M. M. Rams, P. Sierant, O. Dutta, P. Horodecki, and J. Zakrzewski, At the Limits of Criticality-Based Quantum Metrology: Apparent Super-Heisenberg Scaling Revisited, Phys. Rev. X 8, 021022 (2018).

[60] W. L. You, Y. W. Li, and S. J. Gu, Fidelity, dynamic structure factor, and susceptibility in critical phenomena, Phys. Rev. E 76, 022101 (2007).

[61] X. Tan, D.-W. Zhang, Z. Yang, J. Chu, Y.-Q. Zhu, D. Li, X. Yang, S. Song, Z. Han, Z. Li, Y. Dong, H.-F. Yu, H. Yan, S.-L. Zhu, and Y. Yu, Experimental Measurement of the Quantum Metric Tensor and Related Topological Phase Transition with a Superconducting Qubit, Phys. Rev. Lett. 122, 210401 (2019).

[62] M. Yu, P. Yang, M. Gong, Q. Cao, Q. Lu, H. Liu, S. Zhang, M. B. Plenio, F. Jelezko, T. Ozawa, N. Goldman, and J. Cai,
Experimental measurement of the quantum geometric tensor using coupled qubits in diamond, Natl. Sci. Rev. 7, 254 (2020).

[63] A. Gianfrate, O. Bleu, L. Dominici, V. Ardizzone, M. De Giorgi, D. Ballarini, K. West, L. N. Pfeiffer, D. D. Solnyshkov, D. Sanvitto, and G. Malpuech, Measurement of the quantum geometric tensor and of the anomalous Hall drift, Nature (London) 578, 381 (2020).

[64] M. Miyaji, T. Numasawa, N. Shiba, T. Takayanagi, and K. Watanabe, Distance between Quantum States and GaugeGravity Duality, Phys. Rev. Lett. 115, 261602 (2015).

[65] D. Braun, G. Adesso, F. Benatti, R. Floreanini, U. Marzolino, M. W. Mitchell, and S. Pirandola, Quantum-enhanced measurements without entanglement, Rev. Mod. Phys. 90, 035006 (2018).

[66] P. Buonsante and A. Vezzani, Ground-State Fidelity and Bipartite Entanglement in the Bose-Hubbard Model, Phys. Rev. Lett. 98, 110601 (2007).

[67] W.-L. You, C.-J. Zhang, W. Ni, M. Gong, and A. M. Oleś, Emergent phases in a compass chain with multisite interactions, Phys. Rev. B 95, 224404 (2017).

[68] W.-L. You and L. He, Generalized fidelity susceptibility at phase transitions, J. Phys.: Condens. Matter 27, 205601 (2015).

[69] S. R. White, Density-matrix algorithms for quantum renormalization groups, Phys. Rev. B 48, 10345 (1993).

[70] U. Schollwöck, The density-matrix renormalization group, Rev. Mod. Phys. 77, 259 (2005).

[71] U. Schollwöck, The density-matrix renormalization group in the age of matrix product states, Ann. Phys. (NY) 326, 96 (2011).

[72] M. E. Fisher and M. N. Barber, Scaling Theory for Finite-Size Effects in the Critical Region, Phys. Rev. Lett. 28, 1516 (1972).

[73] H.-J. Mikeska and A. K. Kolezhuk, One-dimensional magnetism, in Quantum Magnetism, edited by U. Schollwöck, J. Richter, D. J. J. Farnell, and R. F. Bishop, Lecture Notes in Physics Vol. 645 (Springer, Berlin, 2008), pp. 1-83.

[74] T.-C. Yi, W.-L. You, N. Wu, and A. M. Oleś, Criticality and factorization in the Heisenberg chain with Dzyaloshinskii-Moriya interaction, Phys. Rev. B 100, 024423 (2019).

[75] H. Ueda, H. Nakano, and K. Kusakabe, Finite-size scaling of string order parameters characterizing the Haldane phase, Phys. Rev. B 78, 224402 (2008). 